\title{
Solutions of the Dirac-Fock equations without projector
}

\author{
Éric PATUREL
}

\begin{abstract}
In this paper we prove the existence of infinitely many solutions of the Dirac-Fock equations with $N$ electrons turning around a nucleus of atomic charge $Z$, satisfying $N<Z+1$ and $\alpha \max (Z, N)<\frac{2}{\frac{2}{\pi}+\frac{\pi}{2}}$, where $\alpha \approx \frac{1}{137}$ is the fundamental constant of the electromagnetic interaction. This work is an improvement of an article of Esteban-Séré, where the same result was proved under more restrictive assumptions on $N$.
\end{abstract}

\section{Introduction}

The main topic of this talk is the Dirac-Fock (DF) model. Many quantum models have been studied from a mathematical point of view during this last fifty years, for example the Hartree $(\mathrm{H})$ model, and its more realistic variant, called Hartree-Fock (HF). These studies led to many results, for example the existence of a ground state [7], of infinitely many stationary states [8] for the HF model. This latter is moreover very useful in computational chemistry, where it yields results on the energy levels of atoms, that agree with experimental data, as long as the atomic charge is not too large. The HF model is nevertheless based on a nonrelativistic equation, involving the stationary Schrödinger operator $-\Delta$. In 1935, Swirles introduced the DiracFock energy functional [10]: this functional approximates the relativistic energy of a system of $N$ electrons moving around a static nucleus of large positive charge $Z$. There remains some strong approximations: the nucleus is supposed to be fixed (Born-Oppenheimer approximation), the only interactions considered are electrostatic (no magnetic - or spin - interaction), and the wave function of $N$ electrons is represented by a Slater determinant. However, the numerical results obtained with the DF model are in very good agreement with experimental data (see [6], [5], [3]).

MSC 2000: 81Qxx, 58Exx, 81Vxx

Keywords : variational methods, strongly indefinite functional, Lyapunov-Schmidt reduction, Conley-Zehnder index, relativistic quantum mechanics, quantum chemistry 
In [4], M.J. Esteban and E. Séré proved the existence of infinitely many stationary states for the DF model, provided $N$ and $Z$ satisfy the following conditions:

$$
N<Z+1, \alpha \max (Z, 3 N-1)<\frac{2}{\frac{2}{\pi}+\frac{\pi}{2}}:=\alpha Z_{c} .
$$

Here $\alpha \approx \frac{1}{137}$ stands for the fundamental constant of the electromagnetic interaction. Their method is variational and, after changes in the functional (smoothing the potential and penalizing the constraint), they reduce it thanks to a concavity property: the major advantage is that the new functional is no more strongly indefinite. Once this reduction is made, they build min-max sequences by a linking argument. Unfortunately, the concavity argument does not work for $\alpha(3 N-1)>\alpha Z_{c}$.

Our aim is to improve this result, so that it becomes true for every $N$ and $Z$ satisfying

$$
N<Z+1, \alpha \max (Z, N)<\alpha Z_{c} .
$$

First note that the result presented here as [4] includes also the case of molecules, involving a distribution of positive charge $Z \mu$, with a probability measure $\mu$, and $Z$ the total positive charge. In the following, we will only consider for convenience a point-like nucleus (the molecular case easily follows).

To set out our result, we first recall the definitions. We will use the system of units such that $\hbar=c=1$, and the mass $m_{e}$ of the electron is also normalized to 1 . The state of a free electron is represented by a wave function $\Psi(t, x)$, with $\Psi(t,.) \in L^{2}\left(\mathbb{R}^{3}, \mathbb{C}^{4}\right)$ for any $t$, which satisfies the free Dirac equation:

$$
i \partial_{t} \Psi=H_{0} \Psi, \text { with } H_{0}=-i \sum_{k=1}^{3} \alpha_{k} \partial_{k}+\beta .
$$

We denote by $(\phi, \psi)_{L^{2}}$ the usual Hermitian product in $L^{2}\left(\mathbb{R}^{3}, \mathbb{C}^{4}\right)$. The Dirac equation contains the $4 \times 4$ complex matrices $\alpha_{1}, \alpha_{2}, \alpha_{3}$ and $\beta$, which satisfy some algebraic conditions ensuring that $H_{0}$ is a symmetric operator satisfying

$$
H_{0}^{2}=-\Delta+1
$$

The following lemma contains, for the reader's convenience, some properties of $H_{0}$ and the coulombic potential $V(x)=\frac{1}{|x|}$, whose proof can be found in [1], [12], [11].

Lemma 1.1. $H_{0}$ is a self-adjoint operator on $L^{2}\left(\mathbb{R}^{3}, \mathbb{C}^{4}\right)$, with domain $H^{1}\left(\mathbb{R}^{3}, \mathbb{C}^{4}\right)$. Its spectrum is $(-\infty,-1] \cup[1,+\infty)$. There are two orthogonal projectors on $L^{2}\left(\mathbb{R}^{3}\right.$, $\left.\mathbb{C}^{4}\right), \Lambda^{+}$and $\Lambda^{-}=1_{L^{2}}-\Lambda^{+}$, both with infinite rank, and such that

$$
\left\{\begin{array}{l}
H_{0} \Lambda^{+}=\Lambda^{+} H_{0}=\sqrt{1-\Delta} \Lambda^{+}=\Lambda^{+} \sqrt{1-\Delta} \\
H_{0} \Lambda^{-}=\Lambda^{-} H_{0}=-\sqrt{1-\Delta} \Lambda^{-}=-\Lambda^{-} \sqrt{1-\Delta}
\end{array}\right.
$$

The coulombic potential $V(x)=\frac{1}{|x|}$ satisfies the following Hardy-type inequalities:

$$
(\varphi, V \varphi)_{L^{2}} \leq \frac{1}{2}\left(\frac{\pi}{2}+\frac{2}{\pi}\right)\left(\varphi,\left|H_{0}\right| \varphi\right)_{L^{2}}
$$


for all $\varphi \in \Lambda^{+}\left(H^{\frac{1}{2}}\right) \cup \Lambda^{-}\left(H^{\frac{1}{2}}\right)$. Moreover,

$$
\begin{aligned}
(\varphi, V \varphi)_{L^{2}} & \leq \frac{\pi}{2}\left(\varphi,\left|H_{0}\right| \varphi\right)_{L^{2}}, \forall \varphi \in H^{\frac{1}{2}} \\
\|V \varphi\|_{L^{2}} & \leq 2\|\nabla \varphi\|_{L^{2}}, \forall \varphi \in H^{1} .
\end{aligned}
$$

We now introduce the functional space of the variational problem associated to the DF model. Let

$$
E=H^{\frac{1}{2}}\left(\mathbb{R}^{3}, \mathbb{C}^{4}\right), E^{+}=\Lambda^{+} E, E^{-}=\Lambda^{-} E .
$$

$E$ is a Hilbert space with Hermitian product

$$
(\varphi, \psi)_{E}=(\varphi, \sqrt{1-\Delta} \psi)_{L^{2}}=\left(\varphi^{+}, \psi^{+}\right)_{E}+\left(\varphi^{-}, \psi^{-}\right)_{E} .
$$

The DF energy functional, denoted by $\mathcal{E}$, is defined on $E^{N}$, with

$$
\begin{aligned}
\mathcal{E}(\Phi)= & \sum_{l=1}^{N}\left(\varphi_{l}, H_{0} \varphi_{l}\right)_{L^{2}}-\alpha Z \sum_{l=1}^{N}\left(\varphi_{l}, V \varphi_{l}\right)_{L^{2}} \\
& +\frac{\alpha}{2} \iint_{\mathbb{R}^{3} \times \mathbb{R}^{3}} V(x-y)[\rho(x) \rho(y)-\operatorname{tr}(R(x, y) R(y, x))] d^{3} x d^{3} y,
\end{aligned}
$$

where $\alpha$ is positive, with physical value $\alpha \approx \frac{1}{137}, \rho$ is a scalar and $R$ is a $4 \times 4$ complex matrix, given by

$$
\rho(x)=\sum_{l=1}^{N}\left(\varphi_{l}(x), \varphi_{l}(x)\right), R(x, y)=\sum_{l=1}^{N} \varphi_{l}(x) \otimes \varphi_{l}^{*}(y) .
$$

Physically, $\rho$ is the electronic density, $R$ is the exchange matrix.

We look for critical points of $\mathcal{E}$ under the normalization constraint

$$
\Phi \in \Sigma=\left\{\Phi \in E^{N} \mid \operatorname{Gram}_{L^{2}} \Phi=\mathbb{1}\right\} .
$$

The manifold $\Sigma$ and the DF functional are invariant under the action of the group $\mathcal{U}(N)$ of $N \times N$ self-adjoint complex matrices with determinant of modulus 1. Thanks to this invariance, we may diagonalize the Lagrange multiplier matrix and write the Euler-Lagrange equations in the following form, called Dirac-Fock equations

$$
\bar{H}_{\Phi} \varphi_{k}=\varepsilon_{k} \varphi_{k}
$$

where

$$
\bar{H}_{\Phi} \psi=H_{0} \psi-\alpha Z V \psi+\alpha(\rho * V) \psi-\alpha \int_{\mathbb{R}^{3}} R(x, y) \psi(y) V(x-y) d y .
$$

Physically, $\bar{H}_{\Phi}$ represents the Hamiltonian of an electron in the mean field due to the nuclei and the electrons. The eigenvalues $\varepsilon_{1}, \ldots, \varepsilon_{N}$ are the energies of each electron in this mean field. With the unit convention, the physically interesting states correspond to $0<\varepsilon_{k}<1$ : a positive energy less than the rest mass of the electron.

Our result is an extension of [4, Theorem 1.2]. We only give here a sketch of its proof, which may be found extensively in [9]: 
Theorem 1.2. Assume that $\alpha \max (Z, N)<\frac{2}{\frac{2}{\pi}+\frac{\pi}{2}}, N<Z+1$, with $Z>0$ the nuclear charge. Then there exists a sequence $\left(\Phi^{n}\right)$ of critical points for the DF functional $\mathcal{E}$ on $\Sigma$. The functions $\varphi_{1}^{n}, \ldots, \varphi_{N}^{n}$ satisfy the normalization constraints $\operatorname{Gram}_{L^{2}} \Phi^{n}=11$. They are smooth outside 0, decay exponentially as well as their derivatives as $|x| \rightarrow+\infty$, and are strong solutions, in $W^{1, \frac{3}{2}}\left(\mathbb{R}^{3}, \mathbb{C}^{4}\right)$, of the DiracFock equations

$$
\bar{H}_{\Phi^{n}} \varphi_{k}^{n}=\varepsilon_{k}^{n} \varphi_{k}^{n}, 1 \leq k \leq N
$$

with

$$
\forall k, 0<\varepsilon_{k}^{n}<1 .
$$

Moreover, as $n$ goes to infinity, the energies $\varepsilon_{k}^{n}$ go to 1 .

Remark 1.3. The corresponding physical bound for $N$ and $Z$ is 124 , with $N \leq Z$. This covers all known atoms and positive ions, natural or artificial.

\section{Sketch of the proof}

In order to prove this result, we are faced with many difficulties: the corresponding functional is strongly indefinite, there is no compactness property available for this functional, the coulombic potential is hard to deal with, and we need a control of the Lagrange multipliers. To avoid successively these obstacles, we use here many approximation tools so that the final variational problem is simpler to handle with. The strategy can be described like this:

(a) In order to get rid of the roughness of the coulombic potential $V$, we replace it with an approximate one, actually a convolution of $V$ with a normalized gaussian depending on a parameter $\nu$. Moreover, we define a problem with penalization of the constraint. The choice of the penalization gives directly a lower bound for the energy of the electrons. This step is exactly the same as in $[4]$.

(b) To get some compactness properties, we change the problem into a periodic one: we consider a functional space of periodic functions. We have to change one more time the shape of the potential. The new functional satisfies the Palais-Smale compactness property.

(c) The last problem is that the functional is still strongly indefinite. To deal with this, we operate a Lyapunov-Schmidt reduction to a finite dimensional problem. This reduction is made possible because of the compactness properties of the functional, once steps (a) and (b) are fulfilled. Such a tool was used in a famous paper by Conley and Zehnder [2] on Arnold's conjecture.

(d) We develop a linking method in the finite dimensional case. This method is based on the construction of a pseudogradient vector field. The LyapunovSchmidt reduction gives then critical points for the periodic problem (b). We have then to translate the estimates on the (Conley-Zehnder) Morse index of the critical points for the finite dimensional problem onto an estimate on the eigenvalues $\varepsilon_{k}$. 
(e) We pass to the limit as the length of the period goes to infinity. There remains to take the limit of the solutions as the penalization parameter $p$ and the smoothness constant $\nu$ go respectively to infinity and 0 : we will use again the same arguments as in [4].

\subsection{First reduction}

Recall our variational formulation:

Problem 2.1. Find critical points for the functional

$$
\begin{aligned}
\mathcal{E}(\Phi)= & \sum_{l=1}^{N}\left(\varphi_{l}, H_{0} \varphi_{l}\right)_{L^{2}}-\alpha Z \sum_{l=1}^{N}\left(\varphi_{l}, V \varphi_{l}\right)_{L^{2}} \\
& +\frac{\alpha}{2} \iint_{\mathbb{R}^{3} \times \mathbb{R}^{3}} V(x-y)[\rho(x) \rho(y)-\operatorname{tr}(R(x, y) R(y, x))] d^{3} x d^{3} y,
\end{aligned}
$$

under the constraint

$$
\Phi \in \Sigma=\left\{\Phi \in E^{N} \mid \operatorname{Gram}_{L^{2}} \Phi=\mathbb{1}\right\}
$$

with the following condition on the matrix of Lagrange multipliers $\Lambda$,

$$
\left\{\begin{array}{l}
\exists u \in \mathcal{U}(N), u \Lambda u^{*}=\operatorname{Diag}\left(\varepsilon_{1}, \ldots, \varepsilon_{N}\right) \\
\text { with } \forall k, 0<\varepsilon_{k}<1
\end{array}\right.
$$

The first transformation of Problem 2.1 into a more practical one was introduced in [4] (to which we refer for more details): as the coulombic potential $V$ is not a compact perturbation of $H_{0}$, we replace it with a regularized potential, for $\nu>0$ :

$$
V_{\nu}(x)=\left(g_{\nu} * V\right)(x), \text { with } g_{\nu}(x)=\frac{1}{(2 \pi \nu)^{\frac{3}{2}}} e^{-\frac{|x|^{2}}{2 \nu}}
$$

This replacement is made for all terms involving $V$, i.e. attractive potential and electronic repulsion and exchange terms. We denote by $\mathcal{E}_{\nu}$ the corresponding functional, and the associated one-particle Hamiltonian is denoted by $\bar{H}_{\Phi}^{\nu}$.

As announced in (a), we also replace the constraint " $\Phi \in \Sigma$ " by a penalization term $\pi_{p}$ depending on an integer $p$. We put

$$
\pi_{p}(\Phi)=\operatorname{tr}\left[\left(\operatorname{Gram}_{L^{2}} \Phi\right)^{p}\left(\mathbb{1}-\operatorname{Gram}_{L^{2}} \Phi\right)^{-1}\right],
$$

and we define the penalized functional

$$
\mathcal{F}_{\nu, p}(\Phi)=\mathcal{E}_{\nu}(\Phi)-\pi_{p}(\Phi), \text { for } \Phi \in A=\left\{\Phi \in E^{N} \mid 0<\operatorname{Gram}_{L^{2}} \Phi<\mathbb{1}\right\} .
$$

Note that $\mathcal{F}_{\nu, p}$ is invariant under the action of the group $\mathcal{U}(N)$, denoted by a dot. Any $\mathcal{U}(N)$ orbit in $A$ contains a point $\Phi$ such that $\operatorname{Gram}_{L^{2}} \Phi$ is diagonal, with eigenvalues in nondecreasing order:

$$
\operatorname{Gram}_{L^{2}} \Phi=\operatorname{Diag}\left(\sigma_{1}, \ldots, \sigma_{N}\right), 0<\sigma_{1} \leq \ldots \leq \sigma_{N}<1 .
$$


We call $\mathcal{O}$ the set of points of $A$ satisfying (2.5). If $\Phi \in \mathcal{O}$, then

$$
\frac{\partial \mathcal{F}_{\nu, p}}{\partial \varphi_{k}}(\Phi)=\bar{H}_{\Phi}^{\nu} \varphi_{k}-\varepsilon_{k} \varphi_{k}
$$

with

$$
\varepsilon_{k}=e_{p}\left(\sigma_{k}\right), e_{p}(x)=\frac{d}{d x}\left(\frac{x^{p}}{1-x}\right)=\frac{p x^{p-1}-(p-1) x^{p}}{(1-x)^{2}} .
$$

The advantage of this penalization is that $e_{p}$ is a positive increasing function on $(0,1)$, so that $0<\varepsilon_{1} \leq \ldots \leq \varepsilon_{N}$. Hence, the critical points of $\mathcal{F}_{\nu, p}$ in $\mathcal{O}$ are solutions of a nonlinear eigenvalue problem with positive eigenvalues. So we may define the following problem

Problem 2.2. Find critical points $\Phi$ of the functional $\mathcal{F}_{\nu, p}$ on the domain $A$, satisfying moreover:

$$
\left\{\begin{array}{l}
\exists u \in \mathcal{U}(N) \mid \operatorname{Gram}_{L^{2}}(u . \Phi)=\operatorname{Diag}\left(\sigma_{1}, \ldots \sigma_{N}\right) \\
\text { with, for all } k, \varepsilon_{k}=e_{p}\left(\sigma_{k}\right)<1 .
\end{array}\right.
$$

We actually need a control on the critical points for $\mathcal{F}_{\nu, p}$, but also on some of its Palais-Smale sequences. Fortunately, we may use a compactness result [4, Lemma 2.1], which indicates the behaviour some Palais-Smale sequences, and ensures that the solutions of Problem 2.2 we will find actually converge to solutions of Problem 2.1 .

\subsection{Reduction to the periodic setting}

From now on, we consider fixed $\nu \in(0,1)$ and $p>3$. It is possible to proceed to another transformation of the variational problem, as announced in (b). Let $L \in \mathbb{R}$, $L>0$ and $\omega=\frac{2 \pi}{L}$,

$$
E_{\omega}=H_{p e r}^{\frac{1}{2}}\left(\left(-\frac{L}{2}, \frac{L}{2}\right)^{3}, \mathbb{C}^{4}\right),
$$

defined as functions with the Fourier series $\psi(x)=\sum_{p \in \mathbb{Z}^{3}} a_{\omega p} e^{i \omega p \cdot x}$ where the Fourier coefficients $a_{\omega p} \in \mathbb{C}^{4}$ satisfy $\sum_{p \in \mathbb{Z}^{3}}\left(1+|\omega p|^{2}\right)^{\frac{1}{2}}\left|a_{\omega p}\right|^{2}<\infty$. We may define the periodic free Dirac operator acting on $L_{p e r}^{2}\left(\left(-\frac{L}{2}, \frac{L}{2}\right)^{3}, \mathbb{C}^{4}\right)$ with domain $E_{\omega}$ with the same formula (1.1). Since $H_{0}^{2}=-\Delta+1$, the spectrum of this operator is contained in $(-\infty,-1] \cup[1,+\infty)$. This allows to define two orthogonal projectors on $L_{\text {per }}^{2}\left(\left(-\frac{L}{2}, \frac{L}{2}\right)^{3}, \mathbb{C}^{4}\right)$, still denoted by $\Lambda^{+}$and $\Lambda^{-}$, satisfying the same properties (1.3) as their equivalent in the non-periodic case.

We now have to define a periodic potential corresponding to the coulombic potential. Let $G_{\nu, \omega}$ be the periodic potential defined by its Fourier series

$$
G_{\nu, \omega}(x)=\frac{\omega}{4 \pi} \sum_{p \in \mathbb{Z}^{3}, p \neq 0} \frac{g_{\nu}(p) e^{i \omega p . x}}{|p|^{2}} .
$$


Remark that the coefficients are, for $p \neq 0$,

$$
c_{\omega p}=\frac{\omega g_{\nu}(p)}{4 \pi|p|^{2}}=\widehat{V_{\nu}}(p)
$$

This periodic potential is no more positive, but satisfies $G_{\nu, \omega} \geq-\omega C_{0}$ for a fixed $C_{0}>0$. This causes a little change in the computations, relatively to the non-periodic case, but the essential properties are preserved, as $\omega$ is close enough to 0 .

Thanks to the Fourier series computation, we are able to translate in the periodic case the results of Lemma 1.1, in order to get similar estimates on periodic function spaces.

We may define the periodic DF-functional, depending on the parameters $\nu, p$ (fixed) and $\omega$ :

$$
\mathcal{P}_{\omega}(\Phi)=\mathcal{E}_{\nu, \omega}(\Phi)-\pi_{p, \omega}(\Phi)
$$

that is,

$$
\begin{aligned}
\mathcal{P}_{\omega}(\Phi)= & \sum_{k=1}^{N}\left(\varphi_{k}, H_{0} \varphi_{k}\right)_{L_{p e r}^{2}}-\alpha Z \sum_{k=1}^{N}\left(\varphi_{k}, G_{\nu, \omega} \varphi_{k}\right)_{L_{p e r}^{2}} \\
& +\frac{\alpha}{2} \iint_{\left(-\frac{L}{2}, \frac{L}{2}\right)^{3} \times\left(-\frac{L}{2}, \frac{L}{2}\right)^{3}} G_{\nu, \omega}(x-y)[\rho(x) \rho(y)-\operatorname{tr}(R(x, y) R(y, x))] d x d y \\
(2.10) \quad & -\operatorname{tr}\left[\left(\operatorname{Gram}_{L_{p e r}^{2}} \Phi\right)^{p}\left(\mathbb{1}-\operatorname{Gram}_{L_{p e r}^{2}} \Phi\right)^{-1}\right] .
\end{aligned}
$$

This functional is defined in the space

$$
A_{\omega}=\left\{\Phi \in E_{\omega}^{N} \mid 0<\operatorname{Gram}_{L_{p e r}^{2}} \Phi<\mathbb{1}\right\} .
$$

The new functional satisfies the Palais-Smale compactness property. Note that, as in the non-periodic case, this functional is invariant by the action of $\mathcal{U}(N)$. We may then define

Problem 2.3. Find critical points $\Psi$ of the functional $\mathcal{P}_{\omega}$ in the space $A_{\omega}$, with the following property

$$
\left\{\begin{array}{l}
\exists u \in \mathcal{U}(N) \mid \operatorname{Gram}_{L_{\text {per }}^{2}}(u . \Psi)=\operatorname{Diag}\left(\sigma_{1}, \ldots \sigma_{N}\right) \\
\exists b_{0}, \forall k, \varepsilon_{k}=e_{p}\left(\sigma_{k}\right) \leq b_{0}<1
\end{array}\right.
$$

The resolution of this variational framework will give solutions of Problem 2.2 since we get a convergence lemma, which achieves the point (b) of the program.

\subsection{Lyapunov-Schmidt reduction}

So we are led to find critical points for the functional $\mathcal{P}_{\omega}$ corresponding to the periodic problem. Unfortunately, this functional is still strongly indefinite. One way to deal with this problem is to reduce the functional space to a finite dimensional space, thanks to a Lyapunov-Schmidt method. 
This reduction is based on the fact that we may split any wave function $\Phi$ into two components: a "high frequency" component $\Phi_{U}$ and a "low frequency" one $\Phi_{D}$, where frequency is meant to be the (norm of the) eigenvalue for the free Dirac operator $H_{0}$. Thanks to a contraction property of $H_{0}^{-1}$, we solve, using a fixed point theorem, the part of the DF-equations related to the high frequency part: this solution depends of course of the low frequency component.

Let $\tilde{\lambda}>1$. We consider the projection $\Lambda_{U}$ (resp. $\Lambda_{D}$ ) on the eigenspaces for $H_{0}$ corresponding to eigenvalues $\lambda$ with $|\lambda|>\tilde{\lambda}$ (resp. $|\lambda| \leq \tilde{\lambda}$ ). We get of course $H_{0} \Lambda_{U}=\Lambda_{U} H_{0}$, and $H_{0} \Lambda_{D}=\Lambda_{D} H_{0}$. We put $\Phi_{U}=\Lambda_{U} \Phi$, and $\Phi_{D}=\Lambda_{D} \Phi$.

Using the projectors $\Lambda_{U}$ and $\Lambda_{D}$ on the periodic Euler-Lagrange equation, we get the following system:

$$
\left\{\begin{array}{l}
\left(\varphi_{k}\right)_{D}=H_{0}^{-1}\left(\Lambda_{D}\left[M_{\rho} \varphi_{k}+N_{R} \varphi_{k}+\frac{\partial \pi_{p, \omega}}{\partial \varphi_{k}}\right]\right) \\
\left(\varphi_{k}\right)_{U}=H_{0}^{-1}\left(\Lambda_{U}\left[M_{\rho} \varphi_{k}+N_{R} \varphi_{k}+\frac{\partial \pi_{p, \omega}}{\partial \varphi_{k}}\right]\right),
\end{array}\right.
$$

where $M_{\rho}=\alpha Z G_{\nu, \omega}-\alpha\left(\rho * G_{\nu, \omega}\right)$ and $N_{R}$ is the operator

$$
N_{R}(\psi)=\int_{\left(-\frac{L}{2}, \frac{L}{2}\right)^{3}} R(x, y) \psi(y) G_{\nu, \omega}(x-y) d y .
$$

We observe that, given $\bar{\Phi}=\left(\bar{\varphi}_{k}\right) \in \Lambda_{D} E_{\omega}$ for every $k$, a solution to the first equation in 2.13 may be written as $\Xi=\left(\xi_{k}\right)_{1 \leq k \leq N}$, with $\xi_{k} \in \Lambda_{U} E_{\omega}$ for all $k$, and $\Xi$ is a fixed point of the operator $\mathcal{I}_{\bar{\Phi}}$ mapping $\left(\Lambda_{U} E_{\omega}\right)^{N}$ into itself, given by

$$
\Xi \stackrel{\mathcal{I}_{\bar{\Phi}}}{\longrightarrow}\left(H_{0}^{-1}\left[\Lambda_{U}\left(M_{\rho(\bar{\Phi}+\Xi)} \xi_{k}+N_{R(\bar{\Phi}+\Xi)} \xi_{k}+\frac{\partial \pi_{p, \omega}}{\partial \varphi_{k}}(\bar{\Phi}+\Xi)\right)\right]\right)_{1 \leq k \leq N} .
$$

The next lemma shows that we may apply the Banach fixed point theorem to find an unique $\bar{\Xi}$ associated to $\bar{\Phi}$. The following estimate will depend on $\nu$, and we check that the considered $\bar{\Phi}+\bar{\Xi}$ is not too close to the boundary $\left\{\Phi \mid \operatorname{Gram}_{L_{\text {per }}^{2}} \Phi=\mathbb{1}\right\}$.

Lemma 2.4. Let $\mu \in(0,1)$. Recall that we have fixed $\nu \in(0,1)$ and $p>3$. There exists $\tilde{\lambda}>0$ such that, given any $\lambda \geq \tilde{\lambda}$ and $\bar{\Phi} \in\left(\Lambda_{D} E_{\omega}\right)^{N}$ satisfying $0<$ $\operatorname{Gram}_{L_{\text {per }}^{2}} \Phi<(1-\mu) \mathbb{1}$, (where the projections $\Lambda_{D}$ and $\Lambda_{U}$ are defined with $\lambda$ ), the application $\mathcal{I}_{\bar{\Phi}}$ defined above, restricted to a ball of radius $\frac{\mu}{2}$ around 0 , is contracting. Moreover, taking $\tilde{\lambda}$ large enough, the ball $\left(\Lambda_{U}\left(E_{\omega}\right)\right)^{N} \cap B_{\left(L_{p e r}^{2}\right)^{N}}\left(0, \frac{\mu}{2}\right)$ is globally invariant relatively to the action of $\mathcal{I}_{\bar{\Phi}}$.

Then the application of the Banach fixed point theorem gives a fixed point for $\mathcal{I}_{\bar{\Phi}}$ in the ball $\left(\Lambda_{U}\left(E_{\omega}\right)\right)^{N} \cap B_{\left(L_{p e r}^{2}\right)^{N}}\left(0, \frac{\mu}{2}\right)$. Thus we may define the map $h_{\tilde{\lambda}}$ from $\left(\Lambda_{D} E_{\omega}\right)^{N}$ to $\left(\Lambda_{U} E_{\omega}\right)^{N} \cap B_{\left(L_{p e r}^{2}\right)}\left(0, \frac{\mu}{2}\right)$, associating to every $\bar{\Phi}$ the unique fixed point of $\mathcal{I}_{\bar{\Phi}}$ in the ball. We put $\bar{\Xi}=h_{\tilde{\lambda}}(\bar{\Phi})$.

Thanks to this property, we can reduce our problem to a finite dimensional one: if we put, for $\Phi \in\left(\Lambda_{D} E_{\omega}\right)^{N}$

$$
\mathcal{Q}_{\omega}(\Phi)=\mathcal{P}_{\omega}\left(\Phi+h_{\tilde{\lambda}}(\Phi)\right),
$$

then Problem 2.3 is solved when the following problem is solved:

Problem 2.5. Find critical points $\Phi \in\left(\Lambda_{D} E_{\omega}\right)^{N}$ of the functional $\mathcal{Q}_{\omega}$, such that $\Psi=\Phi+h_{\tilde{\lambda}}(\Phi)$ satisfies condition (2.12). 


\subsection{End of the proof}

Once this reduction is done, we may use variational tools on the function $\mathcal{Q}_{\omega}$ acting on the finite dimensional space $\left(\Lambda_{D} E_{\omega}\right)^{N}$. Briefly speaking, we build a min-max using a linking property. In particular, we have to avoid critical points corresponding to solutions with $N^{\prime}$ electrons, for $N^{\prime}<N$, i.e. critical points $\Phi$ with a null determinant Gram matrix: this is done by constructing a special pseudo-gradient vector field. We refer to [9] for details concerning this (long!) construction. The result of this construction is the following lemma.

Lemma 2.6. Let $N$ and $Z$ satisfy $\alpha \max (N, Z)<\frac{2}{\frac{\pi}{2}+\frac{2}{\pi}}$, and recall that $\nu$ and $p$ are fixed. Given $\omega>0$ small, there exists $J(\omega) \in \mathbb{N}$, and a sequence $\left(\Psi_{\omega}^{j}\right)_{0 \leq j \leq J(\omega)}$, of critical points of $\mathcal{P}_{\omega}$. These critical points satisfy

$$
0<\operatorname{Gram}_{L_{p e r}^{2}} \Psi_{\omega}^{j}<\mathbb{1}
$$

and there exists a positive sequence $\underline{c}_{j}$ such that, for any $j \geq 0$, we have $0<\underline{c}_{j}<N$, $\lim _{j \rightarrow \infty} \underline{c}_{j}=N$, with

$$
N>\mathcal{P}_{\omega}\left(\Psi_{\omega}^{j}\right) \geq \underline{c}_{j}
$$

Moreover, each $\Psi_{\omega}^{j}$ is of the form

$$
\Psi_{\omega}^{j}=\Phi_{\omega}^{j}+h_{\hat{\lambda}}\left(\Phi_{\omega}^{j}\right)
$$

for some $\hat{\lambda}(\omega, \nu, p)$. The function $\Phi_{\omega}^{j}$ is a critical point of $\mathcal{Q}_{\omega}$, i.e. the reduced functional associated to $\omega$ and $\hat{\lambda}$, and its Morse index $i_{\omega, j}$ satisfies

$$
i_{\omega, j}\left(\Phi_{\omega}^{j}\right) \leq N m(\tilde{\lambda}, \omega)+N^{2}+2 N j,
$$

where $2 N m(\tilde{\lambda}, \omega)$ is the (real) dimension of $\left(\Lambda_{D} E_{\omega}\right)^{N}$.

We remark that, for the construction of linkings of higher order, the functional space needs to have a sufficiently large dimension. Then, the Lyapunov-Schmidt reduction gives a critical point for $\mathcal{P}_{\omega}$ if a critical point for $\mathcal{Q}_{\omega}$ is found. Then we may take advantage of the Morse-type information (2.17) to find an upper bound for the eigenvalues $\varepsilon_{k}$ strictly lower than 1 .

Now, it is possible, thanks to our compactness results, to pass to the limit first as the pulsation $\omega$ goes to 0 : then, $J(\omega) \rightarrow+\infty$ and we obtain a sequence $\left(\tilde{\Psi}^{j}\right)_{j \geq 1}$ of critical points for the functional $\mathcal{F}_{\nu, p}$, with eigenvalues $\tilde{\varepsilon}_{k}^{j}$. Secondly, we may pass to the limit as the parameters $\nu$ and $p$ go respectively to 0 and $\infty$, which solves the initial problem.

Acknowledgement. The author wishes to thank E. Séré for his constant attention and his precious remarks all along this work.

\section{References}

[1] V. I. Burenkov and W. D. Evans. On the evaluation of the norm of an integral operator associated with the stability of one-electron atoms. Proc. Roy. Soc. Edinburgh Sect. A, 128(5):993-1005, 1998. 
[2] C. Conley and E. Zehnder. Morse-type index theory for flows and periodic solutions for Hamiltonian equations. Comm. Pure Appl. Math., 37(2):207-253, 1984 .

cf. p. 4

[3] J. Desclaux. Relativistic Dirac-Fock expectation values for atoms with $Z=1$ to $Z=120$. Atomic Data and Nuclear Data Table, 12:311-406, 1973. cf. p. 1

[4] M. J. Esteban and E. Séré. Solutions of the Dirac-Fock equations for atoms and molecules. Comm. Math. Phys., 203(3):499-530, 1999. cf. p. 2, 3, 4, 5, 6

[5] I.P. Grant. Relativistic Calculation of Atomic Structures. Adv. Phys., 19:747811, 1970.

cf. p. 1

[6] Y.K. Kim. Relativistic self-consistent field theory for closed-shell atoms. Phys. Rev., 154:17-39, 1967.

cf. p. 1

[7] E.H. Lieb and B. Simon. The Hartree-Fock theory for Coulomb systems. Comm. Math. Phys., 53(3):185-194, 1977.

cf. p. 1

[8] P.-L. Lions. Solutions of Hartree-Fock equations for Coulomb systems. Comm. Math. Phys., 109(1):33-97, 1987.

cf. p. 1

[9] E. Paturel. Solutions of the Dirac-Fock equations without projector. Cahiers du Ceremade preprint 9954, mp_arc preprint 99-476, to appear in Annales Henri Poincaré (Birkhäuser). cf. p. 3,9

[10] B. Swirles. The relativistic self-consistent field. Proc. Roy. Soc., A 152:625-649, $1935 . \quad$ cf. p. 1

[11] C. Tix. Lower bound for the ground state energy of the no-pair Hamiltonian. Phys. Lett. B, 405(3-4):293-296, $1997 . \quad$ cf. p. 2

[12] C. Tix. Strict positivity of a relativistic Hamiltonian due to Brown and Ravenhall. Bull. London Math. Soc., 30(3):283-290, 1998.

cf. p. 2

CEREMade, Université Paris IX-Dauphine, Place du Maréchal de Lattre de TAssigny, F-75775 PARis Cedex 16, France

paturel@pi.ceremade.dauphine.fr

www.ceremade.dauphine.fr $/{ }^{\sim}$ paturel 